\title{
Matrine prevents the early development of hepatocellular carcinoma like lesions in rat liver
}

\author{
JIANFEI SHI ${ }^{1}$, XIN HAN $^{1}$, JINFENG WANG ${ }^{1}$, GUANGJIE HAN $^{1}$, MAN ZHAO $^{1}$, XIAOLING DUAN ${ }^{1}$, LILI MI ${ }^{1}$, \\ NING LI ${ }^{1}$, XIAOLEI YIN ${ }^{1}$, HUACUN SHI $^{1}$, CUIZHEN LI ${ }^{1}$, JINTAN GAO $^{2}$, JINSHENG XU $^{3}$ and FEI YIN ${ }^{1}$ \\ ${ }^{1}$ Department of Gastroenterology and Hepatology, The Fourth Hospital of Hebei Medical University, \\ Shijiazhuang, Hebei 050019; ${ }^{2}$ Department of Tuberculosis, Hebei Chest Hospital, Shijiazhuang, Hebei 050041; \\ ${ }^{3}$ Department of Nephrology, The Fourth Hospital of Hebei Medical University, Shijiazhuang, Hebei 050019, P.R. China
}

Received January 12, 2019; Accepted July 5, 2019

DOI: $10.3892 /$ etm.2019.7875

\begin{abstract}
Matrine (C15H24N2O) is an alkaloid extracted from the Chinese herb Sophora flavescens that has anti-fibrotic and anti-cancer properties. The aim of the present study was to determine the chemopreventive effect of matrine on the development of primary hepatocellular carcinoma (HCC) and its possible association with the suppression of the Notch signaling pathway. The rats were randomly divided into four groups: Control, model, low-dose matrine and high-dose matrine groups. The model was established by combining a partial hepatectomy with diethylnitrosamine $(\mathrm{DEN})+2$-acetylaminofluorene (2-AAF). Low- and high-dose matrine groups received intragastric administration of matrine ( 0.25 and $2.5 \mathrm{~g} / \mathrm{l}$ of matrine, respectively). DEN + 2-AAF injections and hepatectomy were not performed in the control group. All rats were sacrificed 2, 4 and 7 weeks after hepatectomy. HCC-like histopathological lesions were detected using hematoxylin and eosin staining. The expression levels of $\alpha$-1-fetoprotein (AFP), albumin (ALB), Notch1 and Hes1 were analyzed using immunohistochemistry. Hepatic lobule structure loss, liver tissue necrosis and inflammatory cell infiltration, and edema degeneration were observed in the model group. By contrast, hepatocyte cord structure was restored and hepatocyte edema degeneration was significantly reduced after 7 weeks of treatment with matrine. In addition, compared with the model group, matrine reduced the expression of AFP, increased the expression of ALB and reduced the expression
\end{abstract}

Correspondence to: Dr Fei Yin, Department of Gastroenterology and Hepatology, The Fourth Hospital of Hebei Medical University, 12 Jiankang Road, Shijiazhuang, Hebei 050019, P.R. China E-mail: yinfei_4y@sina.com

Dr Jinsheng Xu, Department of Nephrology, The Fourth Hospital of Hebei Medical University, 12 Jiankang Road, Shijiazhuang, Hebei 050019, P.R. China

E-mail: xjs5766@126.com

Key words: matrine, hepatocellular carcinoma, $\alpha$-1-fetoprotein, albumin, Notch1, Hes1 of Notch1 and Hes1 (only for high-dose matrine; all $\mathrm{P}<0.05$ ). The findings suggested that matrine could prevent the early development of HCC-like lesions in a rat model, possibly by modulating Notch pathway activation.

\section{Introduction}

Hepatocellular carcinoma ( $\mathrm{HCC}$ ) is the most common type of primary liver cancer. With an age-adjusted incidence of 10.1 cases per 100,000 persons per year, HCC is ranked as the sixth most common neoplasm and the third leading cause of cancer-associated death worldwide (1). In China, HCC is ranked as the fourth most common malignant tumor and the third most deadly disease $(2,3)$. HCC frequently occurs in patients with chronic liver diseases, such as cirrhosis, or following infection of hepatitis B or C virus. Additionally, non-alcoholic fatty liver disease (NAFLD) has been considered another important risk factor for HCC. The association between metabolic syndrome, diabetes, obesity and HCC in NAFLD patients has been supported by retrospective studies (4-7).

Currently, the main therapeutic strategies for $\mathrm{HCC}$ include surgery (tumor resection or liver transplantation), radiation therapy and targeted drug therapy. Sorafenib, an oral multi-kinase inhibitor, was the first drug that was approved for first-line treatment of advanced HCC. Notably, HCC-associated mortality can be prevented by avoiding certain risk factors, e.g. hepatitis B virus vaccination of infants (8) and the prevention of metabolic syndrome through the use of metformin in patients with diabetes or propranolol in patients with hepatitis $\mathrm{C}$ virus-related cirrhosis $(1,9)$. These methods could prevent first primary and second primary recurrence, which is critical to improve the prognosis (10).

In addition to chemically synthesized agents, certain natural products, including coffee, vitamin E and fish oil, as well as phytochemicals, might also reduce the risk of HCC (10). Matrine is an active alkaloid extracted from the dry roots of Sophora flavescens, also known as 'Ku-Shen' in traditional Chinese medicine, which has been used for treatment of various diseases, including HCC and liver fibrosis $(11,12)$. Matrine has proven effective in inhibiting HCC proliferation and migration in vitro, specifically in SMMC-7721 cells (13), 
Huh-7 cells (14) and HepG2 cells (15) and by inhibiting HCC tumor growth in vivo $(15,16)$. The anti-tumor effect of matrine has also been verified in squamous cell carcinomas. Antitumor B, a Chinese herbal mixture of six plants containing matrine, has been demonstrated to inhibit the development of 4-nitroquinoline-1-oxide-induced oral squamous cell carcinomas in mice (17) and lung squamous cell carcinomas in a mouse model (18). However, the majority of these studies emphasize the effect of matrine on an established tumor. The preventive effect of matrine on the formation of HCC tumors remains to be elucidated.

The Notch signaling pathway is a conserved and complex signaling pathway. Its key components include Notch receptor (notch1-4), Notch ligand (delta-like 1, 3, 4, Jagged1 and Jagged2), and target genes Hes and Hey. The Notch signaling pathway has a key role in regulating the development of embryos and organs and controlling cell-fate, including cell proliferation, differentiation, apoptosis, regeneration and other cellular activities (19). Notch deregulation has been indicated to be involved in a number of pathological processes in the liver, including HCC and HCC-accompanied liver fibrosis (20). Notably, matrine inhibits the infiltration of inflammatory monocytes and fibrosis in CCl4-injured livers (21). The inhibitory effect on liver fibrosis was also reported for matrine derivative MD-1 (11). In addition, previous findings revealed that matrine induces hepatic differentiation of hepatic progenitor cells, likely by inhibiting the Notch signaling pathway (22). Therefore, it is important to determine if matrine could prevent the development of primary HCC by suppressing the Notch signaling pathway.

The resistant hepatocyte animal model reflects the early signs of HCC and is commonly used to determine the preventive role of chemical agents in HCC initiation (23). In the present study, a resistant hepatocyte rat model was established by partial hepatectomy combined with treatment of diethylnitrosamine (DEN) and 2-acetylaminofluorene (2-AAF). The model was further used to analyze the effect of matrine on the early changes of liver carcinogenesis.

\section{Materials and methods}

Animals models. A total of 48 male Sprague-Dawley rats (clean grade; 6 weeks old; average weight, $120 \pm 20$ g) were purchased from the Laboratory Animal Center of the Hebei Medical University. The animals were maintained in the National Grade II Experimental Animal Center in a sterile environment (temperature, $22 \pm 1^{\circ} \mathrm{C}$; relative humidity, $50 \pm 1 \%$ and a $12-\mathrm{h}$ light/dark cycle). All animals had access to drinking water and forage. Additionally animals' health and behavior were monitored twice a day at 7:30 a.m. and 6 p.m. by a veterinary officer. In addition, all animal studies were approved and supported by Laboratory Animal Ethics Committee of Fourth Hospital Hebei Medical University (certificate no. 2017026).

The rats were randomly divided into four groups ( $n=12$ /group): Control, model, low-dose matrine and high-dose matrine groups. The model, low-dose matrine and high-dose matrine groups were used to generate preneoplastic lesions for the liver model. Briefly, rats were intraperitoneally injected with $200 \mathrm{mg} / \mathrm{kg}$ DEN (batch no. 1002279831; Sigma-Aldrich; Merck KGaA) just on the first day, then low-dose and high-dose matrine groups received intragastric administration of matrine (batch no. 0301010007160301; Ningxia bauhinia pharmaceutical Co., Ltd.) at a dosage of 1.25 and $12.5 \mathrm{mg} / \mathrm{kg}$ respectively twice per day at 8 a.m. and 5 p.m., which lasted from intraperitoneal injection of DEN to the killing of rats. The interval between two processes was no more than $10 \mathrm{~min}$, so that this experiment can study matrine preventive treatment. While the rats in the model group were given an equal volume of saline solution via gavage. Two weeks after DEN injection, rats received daily intragastric administration of 2-AAF (batch no. 1002176257; Sigma-Aldrich; Merck $\mathrm{KGaA}$ ) at a dosage of $15 \mathrm{mg} / \mathrm{kg}$ for 3 days. Following this, a partial (2/3) hepatectomy was performed. Preoperatively, rats were anesthetized by intraperitoneal injection of 10\% chloral hydrate (batch no. 20161212; Tianjin Damao Chemical Reagent Factory) at a dosage of $300 \mathrm{mg} / \mathrm{kg}$, which was much lower than the LD50 of $480 \mathrm{mg} / \mathrm{kg}$. Three days after the surgery, rats continued to be administered 2-AAF for 1 week. Each rat was weighed before each intragastric administration and given the drug according to its weight to ensure a uniform standard for each administration. In the control group, equivalent abdominal opening/closing surgery and intragastric administration of an equal volume of saline were conducted. However, no DEN injection or 2-AAF supplementation was provided and no hepatectomy was performed in the control group. Partial hepatectomy and intraperitoneal injection of drugs always followed the principle of sterility, and 3 rats died due to postoperative intraperitoneal hemorrhage, which was caused by the surgical ligation suture falling off, but no obvious peritonitis was observed. In this experiment, there were 48 rats in total, 4 rats in each group at each time point. The 3 dead rats were distributed among different experimental groups. In each group, at least 3 rats were still survived at each time point, which met the needs of the experimental statistics $(24,25)$. Each group of rats were sacrificed in 3 batches at 2, 4 and 7 weeks after hepatectomy. At the end of the administration period, animals ate and drank freely for $4 \mathrm{~h}$. The rats were anesthetized by intraperitoneal injection of $10 \%$ chloral hydrate $(300 \mathrm{mg} / \mathrm{kg})$, then they were killed by extirpation of eyeballs and exsanguination (26-28). They were confirmed dead by observation of respiratory and cardiac arrest and the absence of any pain response to needle puncture of the extremities. The livers were then carefully dissected and immediately fixed in $10 \%$ formalin for $24 \mathrm{~h}$ at $4^{\circ} \mathrm{C}$ for subsequent experiments (Fig. 1).

Hematoxylin and eosin $(H \& E)$ staining. Organs were collected, fixed with $10 \%$ formalin and dehydrated using a gradient alcohol series $(60,70,80,90,95$ and $100 \%$ ethanol) followed by two xylene treatments. The samples were embedded in paraffin, then tissues were cut into $5-\mu \mathrm{m}$-thick sections. Tissue sections were subsequently deparaffinized in xylene and rehydrated with a gradient alcohol series (100, 70 and 50\% ethanol) and stained using $\mathrm{H} \& \mathrm{E}$. The sections were stained with hematoxylin staining for $5 \mathrm{~min}$ at $37^{\circ} \mathrm{C}$ and $1 \%$ eosin for $3 \mathrm{~min}$ at $37^{\circ} \mathrm{C}$. All liver sections were observed under a light microscope (magnification, $\mathrm{x} 400$ ).

Immunohistochemistry. Immunohistochemistry was performed as described previously (29). Briefly, hepatic 


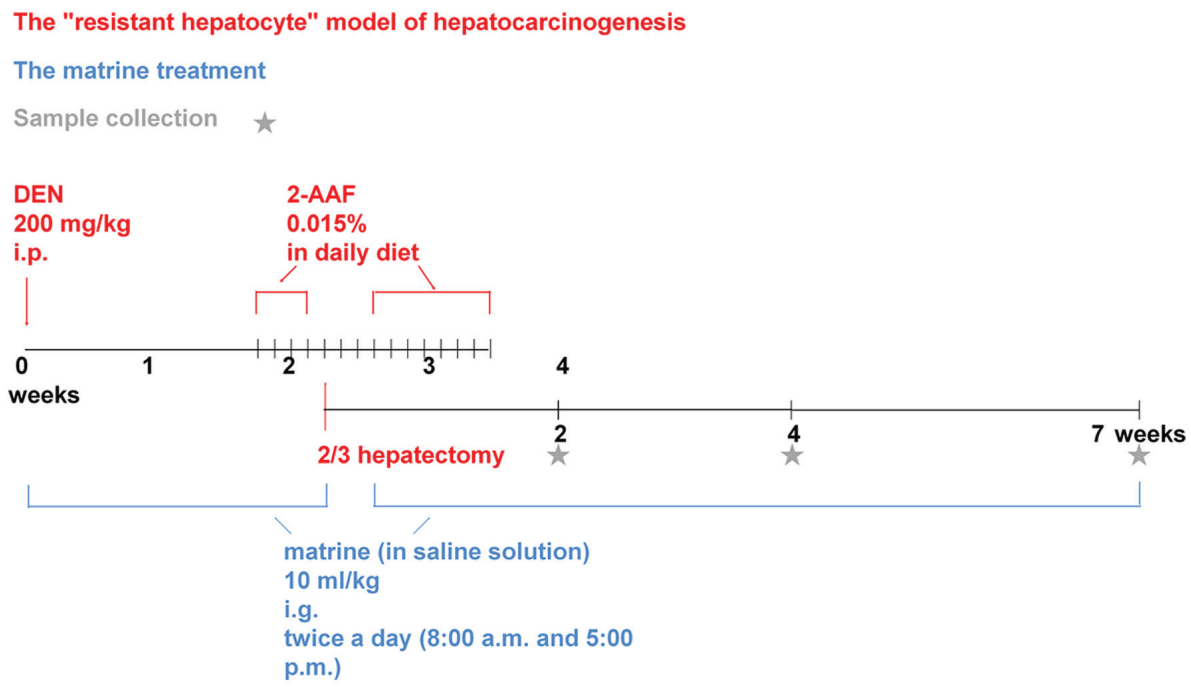

Figure 1. The workflow of animal manipulation of this study. Asterisks indicate sample collection time points following $2 / 3$ hepatectomy. DEN, diethylnitrosamine; 2-AAF, 2-acetylaminofluorene; i.g, intragastric.

tissue was harvested, fixed in $10 \%$ formalin and permeated with paraffin wax. Subsequently, tissues were cut into 6-7- $\mu$ m-thick sections, which was in line with international standards. The sections were then attached to the slides with polylysine membranes and incubated for $4.5 \mathrm{~h}$ at $65^{\circ} \mathrm{C}$. Following this, sections were dewaxed, rehydrated and treated with hydrogen peroxide to block the endogenous peroxidase activity. Slides were incubated with rabbit anti-rat $\alpha$-1-fetoprotein (AFP) polyclonal antibody (cat. no. ab46799; 1:80; Abcam), rabbit anti-rat albumin (ALB) monoclonal antibody (cat. no. ab207327; 1:75; Abcam), rabbit anti-rat Notch1 monoclonal antibody (cat. no. 3608; 1:400; Cell Signaling Technology, Inc.) and rabbit anti-rat Hes1 monoclonal antibody (cat. no. BM4488; 1:100; Wuhan Boster Biological Technology Co., Ltd.). Following this, slides were washed, incubated with a ready-to-use horseradish peroxidase-labeled goat anti-rabbit IgG polymer (cat. no. PV-6001; Beijing Zhongshan Golden Bridge Biotechnology Co., Ltd.) at $37^{\circ} \mathrm{C}$ for $20 \mathrm{~min}$ and then subjected to incubation with diaminobenzidine chromagen at $37^{\circ} \mathrm{C}$ for $20 \mathrm{~min}$. Images were captured using an Olympus BX51T-PHD-J11 light microscope (Olympus Corporation). Immunohistochemical staining was quantified with Image-Pro Plus 5.1 for Windows software (Media Cybernetics, Inc.) using the measurement function. Randomly selected areas were used for analysis by electing 3 slides for each rat and 5 fields for each slide, and combined the number of positive cells with the strong expression of protein and showed the expression intensity of protein as accurately as possible in the form of quantitative data. The number of positive cells and the color intensity of positive cells were obtained, and the immunohistochemical score (IHS) was calculated as follows: A, positive cell number grading: $0-1 \%=0,1-10 \%=1,10-50 \%=2,50-80 \%=3$ and $80-100 \%=4 ; \mathrm{B}$, color intensity of positive cells: Grade 0 (negative), 1 (weak positive), 2 (positive), 3 (strong positive); $\mathrm{IHS}=\mathrm{A} \times \mathrm{B}(30)$.

Statistical analysis. Statistical analyses were performed using SPSS 19.00 (IBM Corp). All data were expressed as mean \pm standard deviation and experiments were repeated at least three times. Data were analyzed using one-way analysis of variance followed by Student-Newman-Keuls post hoc test. $\mathrm{P}<0.05$ was considered to indicate a statistically significant difference.

\section{Results}

Matrine reduces the development of histopathological HCC-like lesions. Normal gross morphology and hepatic lobule structures were indicated in the control group. Furthermore, no significant infiltration of inflammatory cells in the manifold area was observed in the control group (Fig. 2). By contrast, the hepatic lobule structures and the structure of liver cell cords were severely damaged, and the liver cell membrane was damaged 2 weeks after partial hepatectomy in model group. Moreover, edema degeneration was observed in a number of hepatocytes and a large number of inflammatory cells infiltrated the hepatic portal area in model group. A total of 4 weeks after surgery in model group, no significant alterations in liver tissue necrosis or inflammation were observed and injury of hepatic lobule structure was still visible, together with hepatocyte edema and necrosis. However, 7 weeks after surgery, a small proportion of cellular cord structures were detected (Fig. 2).

In the low-dose matrine group, a small proportion of the hepatocyte cord structures were restored 4 weeks after operation. 7 weeks after partial hepatectomy, the hepatocyte edema degeneration was reduced and hepatocyte nodule structures were recovered (Fig. 2). In the high-dose matrine group, a higher protective effect was observed in the week 4 and 7 compared with the model group and low-dose matrine group. Furthermore, discernible lobular structures and a large number of normal liver cell structures were indicated in the high-dose matrine group. In addition, edema degeneration of liver cells and inflammatory cell infiltration were reduced in the high-dose matrine group (Fig. 2).

Matrine reduces the expression of AFP in the liver of resistant hepatocyte rats. AFP is a glycoprotein expressed in hepatoblast 


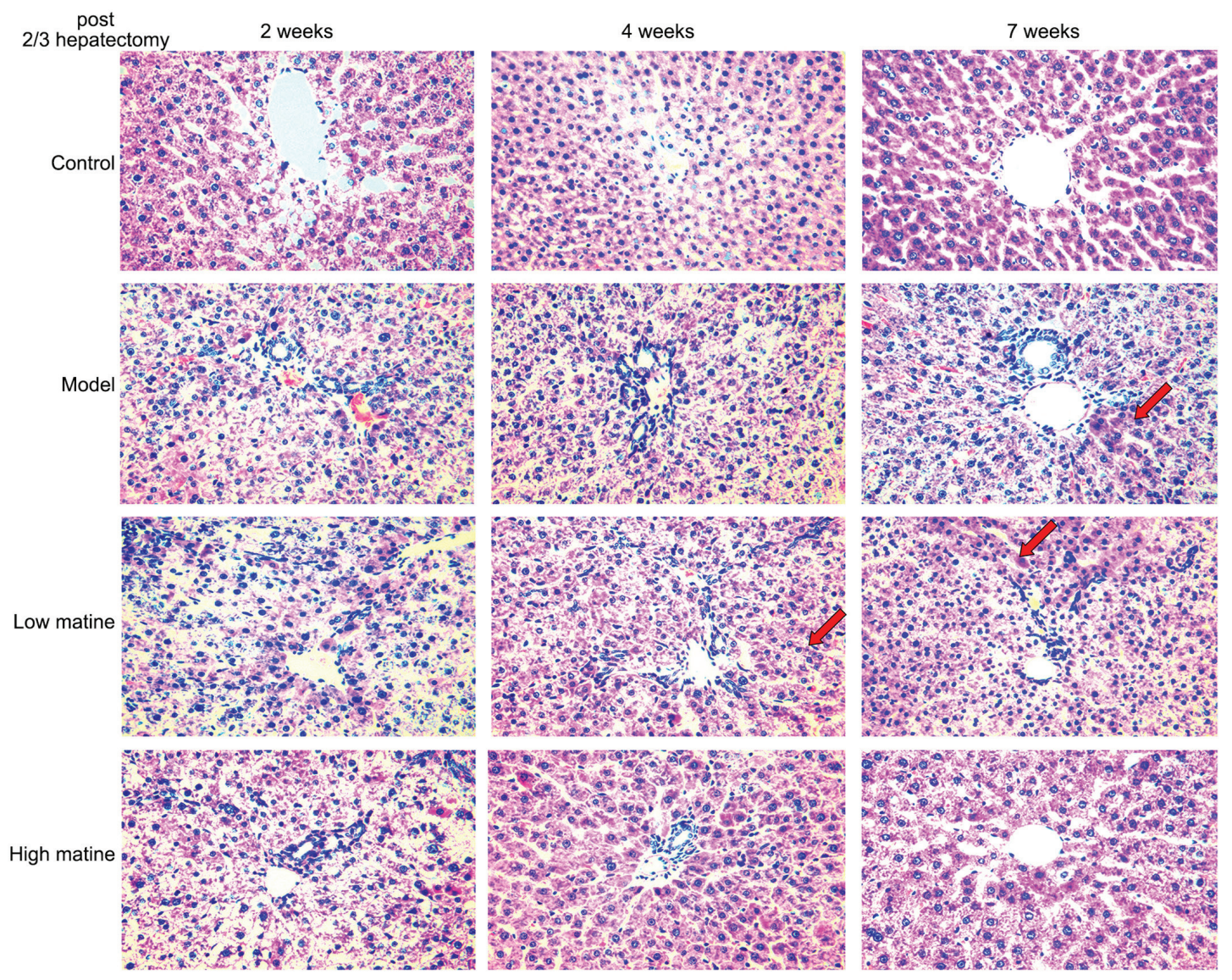

Figure 2. Histopathology of HCC-like lesions in different groups. The livers of rats from the control, model, low-dose matrine and high-dose matrine groups were examined by hematoxylin and eosin staining. Compared with the control group, the model group rats developed HCC-like lesions that exhibited hepatic lobule structure loss, liver tissue necrosis, inflammatory cell infiltration and edema degeneration. Matrine treatment at low or high doses decreased the progression of these HCC-like characteristics. Normal hepatocyte nodule structures were restored (as shown by red arrows). The higher dose of matrine exhibited protection at an earlier (4 weeks) time point compared with low-dose treatment (magnification, x400). HCC, hepatocellular carcinoma.

stem cells, hepatic cancer stem cells and hepatoma cells. When the liver is damaged, an increase of AFP often indicates the severity of damage. The continuous increase of AFP may indicate that part of liver tissue has become cancerous $(31,32)$. According to immunohistochemistry results, AFP was distributed in the cytoplasm and nuclei of liver cells (Fig. 3). No AFP expression was detected in the control group. Notably, AFP expression was significantly upregulated at 2, 4 and 7 weeks after surgery in the model group $(\mathrm{P}<0.01$; Fig. 3 ). In addition, the AFP expression levels in the model groups at 2 and 4 weeks were significantly increased compared with week 7 in the model group $(\mathrm{P}<0.05$ at 2 and 4 weeks; Fig. 3). This indicated that HCC-like lesions occurred at earlier time points.

In the low-dose matrine and high-dose matrine groups, the expression of AFP was significantly decreased at week 2 and 4 compared with the model group; however, it was still increased compared with the control group (low-dose matrine group vs. model group: $\mathrm{P}<0.05$ at 2 weeks and $\mathrm{P}<0.01$ at 4 weeks; high-dose matrine group vs. model group: $\mathrm{P}<0.01$ at 2 weeks and $\mathrm{P}<0.05$ at 4 weeks; Fig. 3). In addition, there was no significant difference in AFP signal between the model group and low-dose or high-dose matrine groups at week 7 (Fig. 3).

Matrine improves the expression of $A L B$ in the liver of resistant hepatocyte rats. ALB is secreted by normal hepatocytes and is often used to distinguish between hepatocytes and hepatoblasts. According to the immunohistochemistry results, ALB was mainly distributed in the cytoplasm of liver cells (Fig. 4). Strong ALB expression was detected in the control group. However, the expression of ALB in the model group was significantly suppressed at week 2, 4 and 7 compared with the control group $(\mathrm{P}<0.05)$. In the low-dose matrine and high-dose matrine groups, significantly increased expression was observed at week 2, 4 and 7 compared with the model group $(\mathrm{P}<0.05)$. In addition, significant differences were found between the low-dose matrine and high-dose matrine groups. Notably, the expression of ALB in the high-dose matrine group was increased compared with in the low-dose matrine group at 4 and 7 weeks (Fig. 4).

Upregulation of Notchl expression in the liver of resistant hepatocyte rats is reduced by matrine treatment. Notch1 is the primary receptor of the Notch signaling pathway and was used as the indicator for the activity of Notch pathway in the present study. According to immunohistochemistry results, Notch1 was primarily distributed in the cytoplasm of liver cells (Fig. 5). No Notch1 expression was detected in the control group. Notably, compared with the control group, Notch1 expression was significantly upregulated at week 2,4 and 7 in the model group $(\mathrm{P}<0.01$ at 2 weeks, $\mathrm{P}<0.01$ at 4 weeks and 

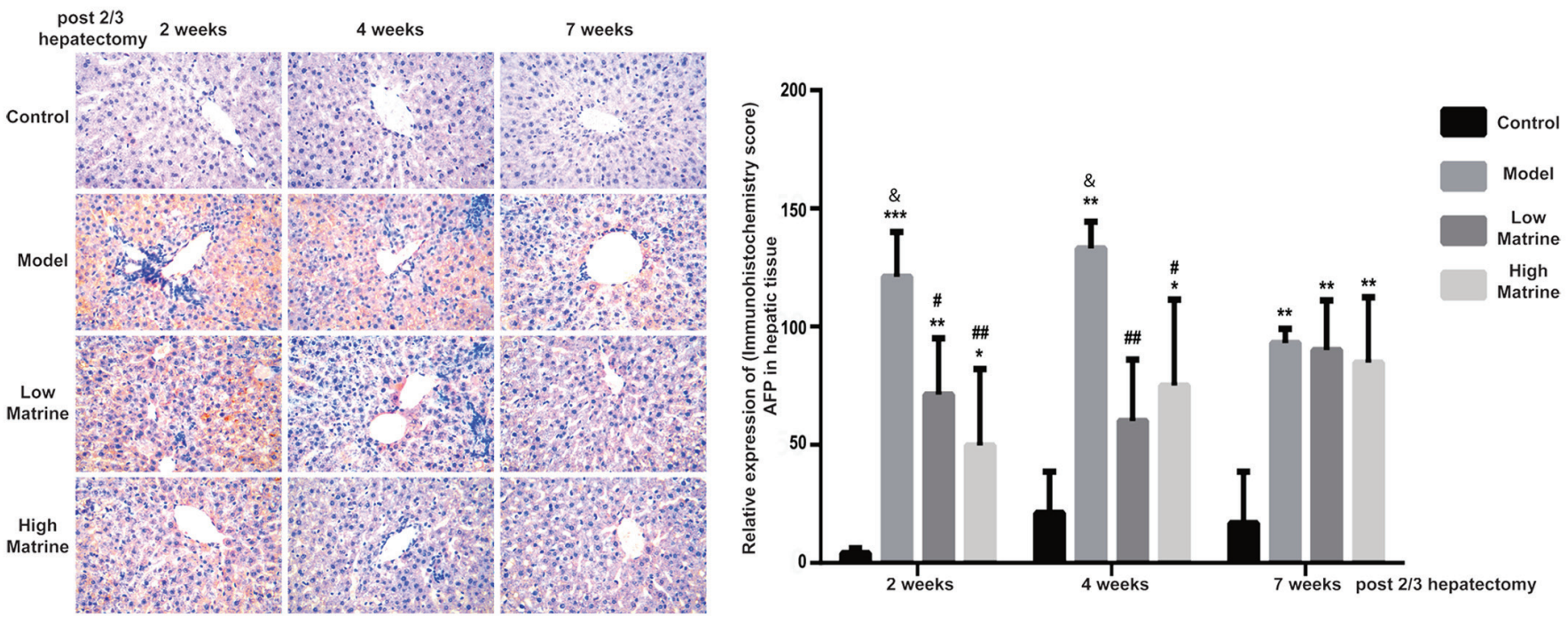

Figure 3. Elevation of AFP expression in the resistant hepatocyte model is reduced by matrine treatment. The expression and distribution of AFP in rat livers from the control, model, low-dose matrine and high-dose matrine groups were examined by immunohistochemistry staining (images in left panel and quantification in right panel). The orange color indicated the presence of AFP. Compared with the control group, the model group rats exhibited elevated AFP distribution. Matrine treatment at low or high doses decreased this elevation at 2 and 4 weeks. Data was presented as the mean \pm standard error of the mean. ${ }^{*} \mathrm{P}<0.05,{ }^{* *} \mathrm{P}<0.01$ and ${ }^{* * *} \mathrm{P}<0.001$ vs. the control group; ${ }^{*} \mathrm{P}<0.05$ and ${ }^{\# \#} \mathrm{P}<0.01$ vs. the model group; ${ }^{\text {}} \mathrm{P}<0.05$ vs. the model group at 7 weeks. Magnification, $\mathrm{x} 400$. AFP, $\alpha$-1-fetoprotein.
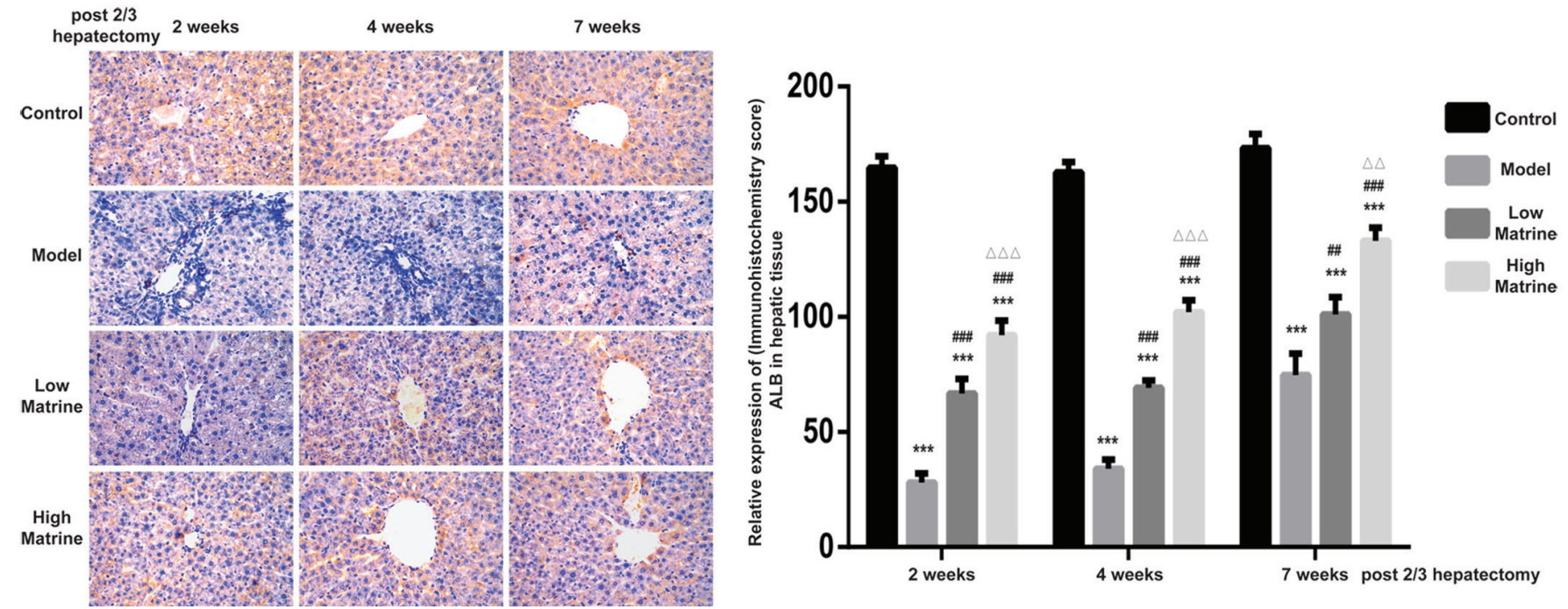

Figure 4. Elevation of ALB expression in the resistant hepatocyte model is enhanced by matrine treatment. The expression and distribution of ALB in rat livers from the control, model, low-dose matrine and high-dose matrine groups were examined by immunohistochemistry (images in left panel and quantification in right panel). The orange color indicated the presence of ALB. Compared with the control group, the model group rats exhibited lower ALB distribution. Matrine treatment at low or high doses enhanced the expression of ALB at 2, 4 and 7 weeks. Data was presented as the mean \pm standard error of the mean. ${ }^{* * * * *} \mathrm{P}<0.001$ vs. the control group; ${ }^{\# \#} \mathrm{P}<0.01$ and ${ }^{\# \# \prime} \mathrm{P}<0.001$ vs. the model group; ${ }^{\Delta \Delta} \mathrm{P}<0.01$ and ${ }^{\Delta \Delta \Delta} \mathrm{P}<0.001$ vs. the low matrine group. Magnification, $\mathrm{x} 400$. ALB, albumin.

$\mathrm{P}<0.001$ at 7 weeks; Fig. 5). This suggested that the HCC-like lesions were accompanied with Notch pathway activation.

In low-dose matrine and high-dose matrine groups, significantly reduced Notch1 expression was observed at week 4 compared with the model group (low-dose matrine group vs. model group at 4 weeks: $\mathrm{P}<0.05$; high-dose matrine vs. model group weeks: $\mathrm{P}<0.01$; Fig. 5); however, no significant differences were found at week 4 between low-dose matrine and high-dose matrine groups. There was no significant difference in Notch1 staining between the model group and either of the matrine groups at 2 and 7 weeks (Fig. 5).
Upregulation of Hesl expression in liver of the resistant hepatocyte model is reduced by matrine treatment. Hes1 is the predominant downstream target gene of the Notch signaling pathway and its expression level was used as the indicator for Notch signaling activity. The present data indicated that Hes1 was primarily distributed in the nuclei of liver cells (Fig. 6). No Hes1 expression was detected in the control group. Notably, compared with the control group, Hes1 expression was significantly upregulated at 2, 4 and 7 weeks after a partial hepatectomy in the model group $(\mathrm{P}<0.001$ at 2 weeks, $\mathrm{P}<0.01$ at 4 weeks and $\mathrm{P}<0.01$ at 7 weeks; Fig. 6). 

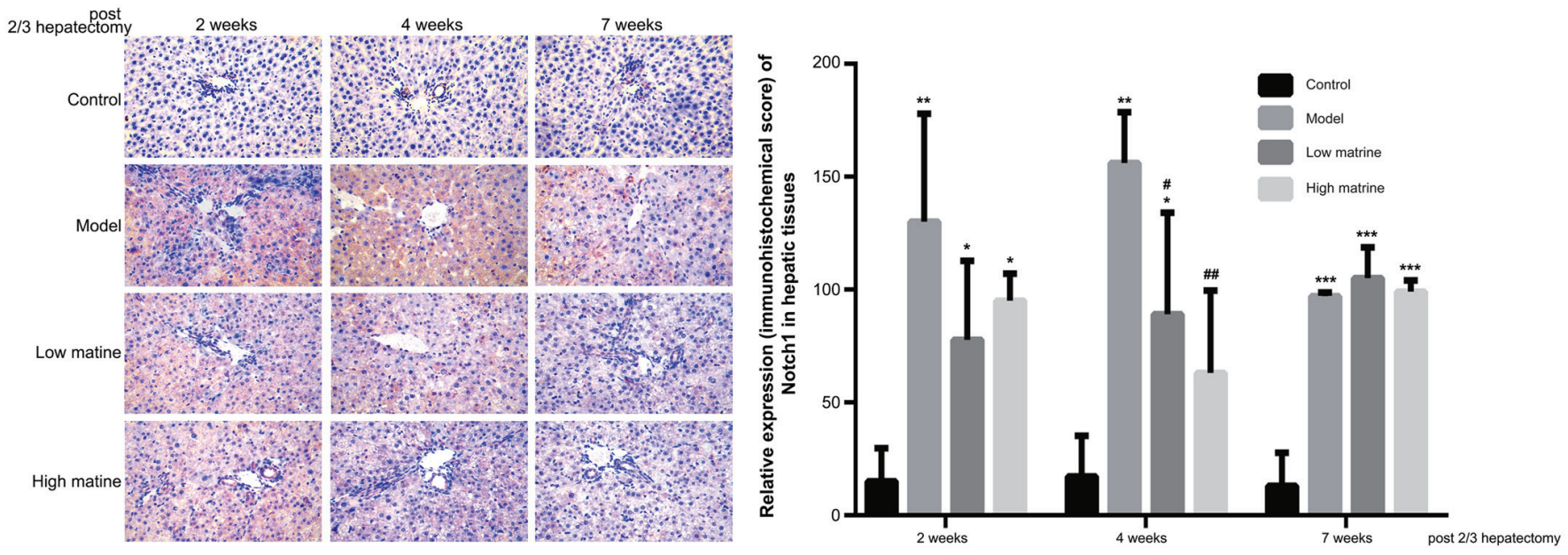

Figure 5. Elevation of Notch1 expression in the resistant hepatocyte model is attenuated by matrine treatment. The expression and distribution of Notch1 in rat livers from the control, model, low-dose matrine and high-dose matrine groups were examined by immunohistochemistry staining (images in left panel and quantification in right panel). The orange color indicated the presence of Notch1. Compared with the control group, the model group exhibited elevated Notch1 distribution. Matrine treatment at low or high doses attenuated this elevation at 2 and 4 weeks. Data was presented as the mean \pm standard error of the mean. ${ }^{*} \mathrm{P}<0.05,{ }^{* *} \mathrm{P}<0.01$ and ${ }^{* * *} \mathrm{P}<0.001$ vs. the control group; ${ }^{*} \mathrm{P}<0.05$ and ${ }^{\# \#} \mathrm{P}<0.01$ vs. the model group; Magnification, $\mathrm{x} 400$.
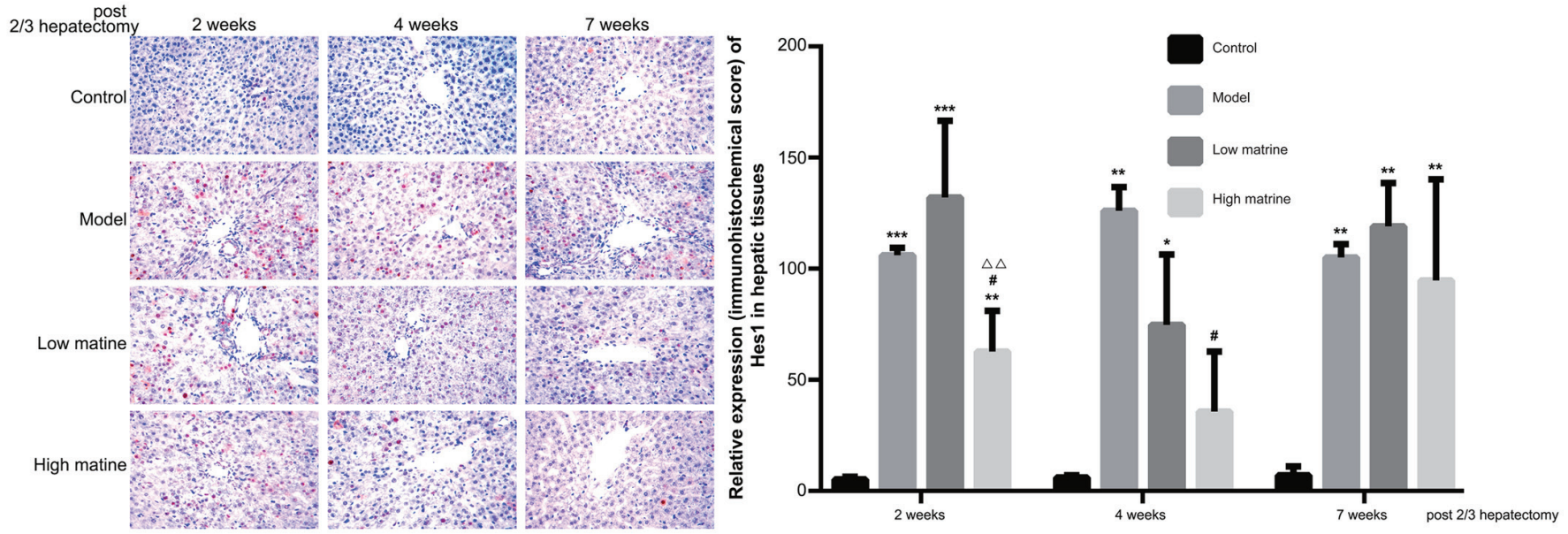

Figure 6. Elevation of Hes1 expression in the resistant hepatocyte model is attenuated by matrine treatment. The expression and distribution of Hes1 in rat livers from the control, model, low-dose matrine and high-dose matrine groups were examined by immunohistochemistry staining (images in left panel and quantification in right panel). The orange color indicated the presence of Hes1. Compared with the control group, the model group rats exhibited elevated Hes1 distribution. High-dose matrine treatment attenuated this elevation at 2 and 4 weeks. Data was presented as the mean \pm standard error of the mean. " $\mathrm{P}<0.05$, ${ }^{* *} \mathrm{P}<0.01$ and ${ }^{* * * *} \mathrm{P}<0.001$ vs. the control group; ${ }^{*} \mathrm{P}<0.05$ vs. the model group; ${ }^{\Delta \Delta} \mathrm{P}<0.01$ vs. the low matrine group. Magnification, $\mathrm{x} 400$.

In the low-dose matrine group, Hes1 expression was not significantly altered compared with the model group. However, in the high-dose matrine group, significantly reduced Hes1 expression was observed at week 2 and 4 compared with the model group ( $\mathrm{P}<0.05$ at 2 weeks and $\mathrm{P}<0.05$ at 4 weeks; Fig. 6). There was no significant difference in Hes1 staining between the model group and either of the matrine groups at 7 weeks (Fig. 6).

\section{Discussion}

Traditional Chinese medicine has been shown to be effective for treating patients with HCC. A number of preclinical studies have indicated that matrine possesses an anti-tumor effect against $\mathrm{HCC}(14,33,34)$. However, it remains to be determined if matrine has a chemopreventive effect against HCC. In this study, a resistant hepatocyte model of hepatocarcinogenesis (initiation with DEN, selection/promotion with 2-AAF and partial hepatectomy) was constructed. This model includes the stimulatory factor associated with tissue regeneration after hepatectomy (33) and reflects the early signs of HCC. Thus, this model is commonly used to determine the preventive role of chemical agents in HCC initiation. For example, this model has been used to demonstrate the chemopreventive effect of folic acid on preneoplastic lesions (35). Similar effects have been observed when applying caffeic acid phenethyl ester (36) and ethanol ethanol extract of Phellinus merrillii (37).

In this study, a resistant hepatocyte rat model was used to explore the chemopreventive effect of matrine on the development of HCC. Compared with the control group, rats in the model group exhibited loss of hepatic lobule structure, signs of damage to structure of liver cell cords and a reduced number 
of liver cells. Moreover, infiltration of inflammatory cells in the hepatic portal area was observed, suggesting the presence of multiple pre-cancerous characteristics. The present results indicated that the liver damage of the low-dose matrine and high-dose matrine groups was mildly alleviated 4 weeks after partial hepatectomy, and was restored at 7 weeks after operating (including the restoration of hepatic cord and lobule structures and reduction of liver cell necrosis) when compared with the model group. Higher restoration was observed in the high-dose matrine group compared with the low-dose matrine group. This was consistent with a previous study, which examined the matrine derivative MD-1 effect on a hepatic fibrosis rat model; their data indicated that fibrosis, necrosis and inflammatory cell infiltration were all restored 4 weeks after modeling (11).

In this experiment, higher AFP expression was detected in the model group when compared with the control group. Furthermore, ALB expression in the model group was reduced when compared with the control group. The high AFP expression suggested that hepatic tissue of the model group exhibited severe damage. Following matrine treatment, the elevated AFP expression after modeling was reduced. Regarding the ALB expression, matrine treatment upregulated the expression when compared with the model group. In addition, the expression of ALB was increased in the high-dose matrine group compared with the low-dose matrine group. The effects of matrine on liver morphology and protein indicated that matrine reduces liver damage and inhibits the initiation of HCC-like alterations. This was consistent with a previous study, which found that matrine derivative MASM may inhibit the proliferation of $\mathrm{HCC}$ in a dose-dependent manner and increase the expression of ALB, indicating that matrine-like drugs could induce the transformation of HCC cells into hepatocytes (12). Similarly, matrine was able to increase the expression of ALB in hepatic oval cells and could induce their differentiation into hepatocytes (22).

Deregulation of the Notch signaling pathway has been reported to be involved in a number pathological processes in the liver, including the development of HCC and HCC-associated liver fibrosis (20). To explore the Notch-related mechanism involved in the preventive effect of matrine, the expression levels of Notch pathway components were examined. Notch1 is the main receptor of the Notch signaling pathway, while Hes1 is the main downstream target gene of the pathway. In the model group, the expression of these two proteins was upregulated, suggesting that the Notch pathway was activated in the HCC-mimicking model. Application of high-dose matrine reduced Notch1 and Hes1 expression, demonstrating that matrine could inhibit the Notch signaling pathway in the HCC-mimicking model. Matrine-induced inhibition of the Notch signaling pathway may alleviate hepatic damage or even reverse hepatic cell carcinogenesis. Liu et al (38) indicated in a model of liver transplantation that upregulation of the Notch signaling pathway could promote differentiation of liver stem cells into hepatofibrotic cells, thereby increasing the secretion of collagen $\mathrm{I} \alpha 1$ and fibrillar junction protein, and aggravating liver damage. However, inhibition of the Notch signaling pathway could reverse liver damage. Similar to the results of the present study, matrine was reported to inhibit the expression of Notch signaling pathway genes in a dose-dependent manner in hepatic oval cells, promoting hepatic oval cell differentiation into hepatocytes $(22,39)$. Notably, Liu et al $(40)$ also found matrine could alleviate myocardial injury and myocardial fibrosis by inhibiting the Notch signaling pathway.

In this experiment, the classic rat Solt-Farber model was used, which is often used to assess acute liver damage and liver cell carcinogenesis. Unfortunately, the typical early-cancer nodules were not induced, indicating that preneoplastic lesions were not completely generated. This might be caused by the inefficient dosage and/or time of the carcinogens used. Dezsö et al (41) also found only regenerative, not neoplastic, growth in rat Solt-Farber experimental hepatocarcinogenesis model. They administered a dosage of $5 \mathrm{mg} / \mathrm{kg} 2-\mathrm{AFF}$ to the rats, while a larger dosage of $15 \mathrm{mg} / \mathrm{kg} 2-\mathrm{AAF}$ was used in the present study, but still did not induce HCC and the present study believes that the Solt-Farber model need to be further improved in the future. However, a model of severe liver damage was made and the application of matrine could alleviate liver damage to a certain extent, which also demonstrated the effect of matrine in alleviating liver inflammation and preventing cancer. In addition, not only has matrine been shown to protect the liver and prevent cancer, but other extracts from the dry roots of Sophora flavescnes, such as oxymatrine, have also been shown to be effective in the treatment of liver disease $(42,43)$. However, there was a lack of multi-center research. As a result of its low cost and low side effects, matrine underwent a lot of research and has potential application in developing countries. In the future, the authors will further study the detailed mechanism of matrine and its derivatives in protecting the liver and preventing cancer.

Taken together, the resistant hepatocyte model in the present study was used to assess the early changes in HCC. Furthermore, the present study aimed to determine the effect of matrine in vivo. The findings indicated that matrine treatment inhibited the development of pre-cancerous characteristics, including changes of hepatic lobule structure and expression changes of AFP and ALB. HCC-associated activation of the Notch signaling pathway was implicated by the expression of Notch1 and Hes1, which was detected in the model and suppressed by matrine treatment. Therefore, it was concluded that matrine may be an effective chemopreventive agent for $\mathrm{HCC}$ and can suppress Notch activation.

\section{Acknowledgements}

Not applicable.

\section{Funding}

The present study was supported by the National Natural Science Foundation of China (grant no. 81072966/H2902), Hebei Provincial Natural Science Foundation of China (grant no. C2011206134) and Youth science and technology programme of Hebei Provincial Health and Family Planning Commission of China (grant no. 20160645).

\section{Availability of data and materials}

The datasets used and/or analyzed during the current study are available from the corresponding author on reasonable request. 


\section{Authors' contributions}

JS, JX and FY carried out the studies, participated in collecting data and drafted the manuscript. XH, JW, GH, MZ, XD, LM, NL, XY, HS, CL and JG performed the experiments and analyzed the data. All authors read and approved the final manuscript.

\section{Ethics approval and consent to participate}

All animal studies were approved and supported by Laboratory Animal Ethics Committee of Fourth Hospital Hebei Medical University.

\section{Patient consent for publication}

Not applicable.

\section{Competing interests}

The authors declare that they have no competing interests.

\section{References}

1. Forner A, Reig M and Bruix J: Hepatocellular carcinoma. Lancet 391: 1301-1314, 2018.

2. Torre LA, Bray F, Siegel RL, Ferlay J, Lortet-Tieulent J and Jemal A: Global cancer statistics, 2012. CA Cancer J Clin 65: 87-108, 2015.

3. Chen W, Zheng R, Baade PD, Zhang S, Zeng H, Bray F, Jemal A, Yu XQ and He J: Cancer statistics in China, 2015. CA Cancer J Clin 66: 115-132, 2016.

4. Chen K, Ma J, Jia X, Ai W, Ma Z and Pan Q: Advancing the understanding of NAFLD to hepatocellular carcinoma development: From experimental models to humans. Biochim Biophys Acta Rev Cancer 1871: 117-125, 2019.

5. Tessitore A, Cicciarelli G, Del Vecchio F, Gaggiano A, Verzella D, Fischietti M, Mastroiaco V, Vetuschi A, Sferra R, Barnabei R, et al: MicroRNA expression analysis in high fat diet-induced NAFLD-NASH-HCC progression: Study on C57BL/6J mice. BMC Cancer 16: 3, 2016.

6. Tian Y, Lyu H, He Y, Xia Y, Li J and Shen F: Comparison of hepatectomy for patients with metabolic syndrome-related $\mathrm{HCC}$ and HBV-related HCC. J Gastrointest Surg 22: 615-623, 2018.

7. Dhar D, Seki E and Karin M: NCOA5, IL-6, type 2 diabetes, and HCC: The deadly quartet. Cell Metab 19: 6-7, 2014.

8. Pazgan-Simon M, Simon KA, Jarowicz E, Rotter K, Szymanek-Pasternak A and Zuwała-Jagiełło J: Hepatitis B virus treatment in hepatocellular carcinoma patients prolongs survival and reduces the risk of cancer recurrence. Clin Exp Hepatol 4 : 210-216, 2018

9. Li WQ, Park Y, McGlynn KA, Hollenbeck AR, Taylor PR, Goldstein AM and Freedman ND: Index-based dietary patterns and risk of incident hepatocellular carcinoma and mortality from chronic liver disease in a prospective study. Hepatology 60 : 588-597, 2014.

10. Singh S, Singh PP, Roberts LR and Sanchez W: Chemopreventive strategies in hepatocellular carcinoma. Nat Rev Gastroenterol Hepatol 11: 45-54, 2014.

11. Feng Y, Ying HY, Qu Y, Cai XB, Xu MY and Lu LG: Novel matrine derivative MD-1 attenuates hepatic fibrosis by inhibiting EGFR activation of hepatic stellate cells. Protein Cell 7: 662-672, 2016.

12. Liu Y, Qi Y, Bai ZH, Ni CX, Ren QH, Xu WH, Xu J, Hu HG, Qiu L, Li JZ, et al: A novel matrine derivate inhibits differentiated human hepatoma cells and hepatic cancer stem-like cells by suppressing PI3K/AKT signaling pathways. Acta Pharmacol Sin 38: 120-132, 2017

13. Gao L, Wang KX, Zhou YZ, Fang JS, Qin XM and Du GH: Uncovering the anticancer mechanism of Compound Kushen Injection against $\mathrm{HCC}$ by integrating quantitative analysis, network analysis and experimental validation. Sci Rep 8: 624, 2018.
14. Wang Y, Zhang S, Liu J, Fang B, Yao J and Cheng B: Matrine inhibits the invasive and migratory properties of human hepatocellular carcinoma by regulating epithelial-mesenchymal transition. Mol Med Rep 18: 911-919, 2018.

15. Zhou H, Xu M, Gao Y, Deng Z, Cao H, Zhang W, Wang Q, Zhang B, Song G, Zhan Y and $\mathrm{Hu}$ T: Matrine induces caspase-independent program cell death in hepatocellular carcinoma through bid-mediated nuclear translocation of apoptosis inducing factor. Mol Cancer 13: 59, 2014.

16. Zhang X and Yu H: Matrine inhibits diethylnitrosamine-induced HCC proliferation in rats through inducing apoptosis via $\mathrm{p} 53$, Bax-dependent caspase-3 activation pathway and down-regulating MLCK overexpression. Iran J Pharm Res 15: 491-499, 2016.

17. Wang Y, Yao R, Gao S, Wen W, Du Y, Szabo E, Hu M, Lubet RA and You M: Chemopreventive effect of a mixture of Chinese Herbs (antitumor B) on chemically induced oral carcinogenesis. Mol Carcinog 52: 49-56, 2013.

18. Wang Y, Zhang Z, Garbow JR, Rowland DJ, Lubet RA, Sit D, Law F and You M: Chemoprevention of lung squamous cell carcinoma in mice by a mixture of Chinese herbs. Cancer Prev Res (Phila) 2: 634-640, 2009.

19. Bigas A and Espinosa L: The multiple usages of Notch signaling in development, cell differentiation and cancer. Curr Opin Cell Biol 55: 1-7, 2018.

20. Ni MM, Wang YR, Wu WW, Xia CC, Zhang YH, Xu J, Xu T and Li J: Novel Insights on Notch signaling pathways in liver fibrosis. Eur J Pharmacol 826: 66-74, 2018.

21. Shi D, Zhang J, Qiu L, Li J, Hu Z and Zhang J: Matrine inhibits infiltration of the inflammatory Gr1(hi) monocyte subset in injured mouse liver through inhibition of monocyte chemoattractant Protein-1. Evid Based Complement Alternat Med 2013: 580673,2013

22. Yang Z, Wang L and Wang X: Matrine induces the hepatic differentiation of WB-F344 rat hepatic progenitor cells and inhibits Jagged 1/HES1 signaling. Mol Med Rep 14: 3841-3847, 2016.

23. Anilkumar TV, Golding M, Edwards RJ, Lalani EN, Sarraf CE and Alison MR: The resistant hepatocyte model of carcinogenesis in the rat: The apparent independent development of oval cell proliferation and early nodules. Carcinogenesis 16: 845-853, 1995.

24. Williams JM, Oh SH, Jorgensen M, Steiger N, Darwiche H, Shupe T and Petersen BE: The role of the wnt family of secreted proteins in rat oval 'Stem' cell-based liver regeneration: Wnt1 drives differentiation. Am J Pathol 176: 2732-2742, 2010.

25. Yovchev MI, Grozdanov PN, Joseph B, Gupta S and Dabeva MD: Novel hepatic progenitor cell surface markers in the adult rat liver. Hepatology 45: 139-149, 2007.

26. Liang K, Zhang J, Yin C, Zhou X and Zhou S: Protective effects and mechanism of TPX2 on neurocyte apoptosis of rats in Alzheimer's disease model. Exp Ther Med 13: 576-580, 2017.

27. Liu JH, Feng D, Zhang YF, Shang Y, Wu Y, Li XF and Pei L: Chloral hydrate preconditioning protects against ischemic stroke via upregulating Annexin A1. CNS Neurosci Ther 21: 718-726, 2015.

28. Zeng X, Zhang L, Sun L, Zhang D, Zhao H, Jia J and Wang W: Recovery from rat sciatic nerve injury in vivo through the use of differentiated MDSCs in vitro. Exp Ther Med 5: 193-196, 2013.

29. Perks WV, Singh RK, Jones GW, Twohig JP, Williams AS, Humphreys IR, Taylor PR, Jones SA and Wang ECY: Death receptor 3 promotes chemokine-directed leukocyte recruitment in acute resolving inflammation and is essential for pathological development of mesothelial fibrosis in chronic disease. Am J Pathol 186: 2813-2823, 2016.

30. Soslow RA, Dannenberg AJ, Rush D, Woerner BM, Khan KN, Masferrer J and Koki AT: COX-2 is expressed in human pulmonary, colonic, and mammary tumors. Cancer 89: 2637-2645, 2000.

31. Li CH, Wang YJ, Dong W, Xiang S, Liang HF, Wang HY, Dong HH, Chen L and Chen XP: Hepatic oval cell lines generate hepatocellular carcinoma following transfection with $\mathrm{HBx}$ gene and treatment with aflatoxin B1 in vivo. Cancer Lett 311: 1-10, 2011.

32. Yovchev MI, Xue Y, Shafritz DA, Locker J and Oertel M: Repopulation of the fibrotic/cirrhotic rat liver by transplanted hepatic stem/progenitor cells and mature hepatocytes. Hepatology 59: 284-295, 2014.

33. Farber E: Cellular biochemistry of the stepwise development of cancer with chemicals: G. H. A. Clowes memorial lecture. Cancer Res 44: 5463-5474, 1984. 
34. Zhang J, Gao Y, Han H, Zou C, Feng Y and Zhang H: Matrine suppresses lung metastasis of human hepatocellular carcinoma by directly targeting matrix metalloproteinase-9. Biochem Biophys Res Commun 515: 57-63, 2019.

35. Chagas CE, Bassoli BK, de Souza CA, Deminice R, Jordão Júnior AA, Paiva SA, Dagli ML, Ong TP and Moreno FS: Folic acid supplementation during early hepatocarcinogenesis: Cellular and molecular effects. Int J Cancer 129: 2073-2082, 2011.

36. Beltran-Ramírez O, Pérez RM, Sierra-Santoyo A and Villa-Trevino S: Cancer prevention mediated by caffeic acid phenethyl ester involves cyp2b1/2 modulation in hepatocarcinogenesis. Toxicol Pathol 40: 466-472, 2012.

37. Yang $\mathrm{CH}$, Chang HY, Chen YC, Lu CC, Huang SS, Huang GJ and Lai HC: Ethanol extract of Phellinus merrillii protects against diethylnitrosamine- and 2-acetylaminofluorene-induced hepatocarcinogenesis in rats. Chin J Integr Med 23: 117-124, 2017.

38. Liu XB, Lo CM, Cheng Q, NG KT, Shao Y, Li CX, Chung SK, $\mathrm{Ng}$ IOL, Yu J and Man K: Oval cells contribute to fibrogenesis of marginal liver grafts under stepwise regulation of aldose reductase and notch signaling. Theranostics 7: 4879-4893, 2017

39. Yang ZY, Wang L, Hou YX and Wang XB: Effects of matrine on oval cellmediated liver regeneration and expression of RBP-JK and HES1. Mol Med Rep 7: 1533-1538, 2013.
40. Liu Z, Wang Y, Zhu H, Qiu C, Guan G, Wang J and Guo Y: Matrine blocks GEs-induced HCSMCs phenotypic conversion via suppressing Dll4-Notch pathway. Eur J Pharmacol 835: 126-131, 2018.

41. Dezsö K, Papp V, Bugyik E, Hegyesi H, Sáfrány G, Bödör C, Nagy P and Paku S: Structural analysis of oval-cell-mediated liver regeneration in rats. Hepatology 56: 1457-1467, 2012

42. Gu YM, Lu JY, Sun W, Jin RM, Ohira T, Zhang Z and Tian XS: Oxymatrine and its metabolite matrine contribute to the hepatotoxicity induced by radix Sophorae tonkinensis in mice. Exp Ther Med 17: 2519-2528, 2019.

43. Jia Y, Long S, Jiang N, Shan Z, Lu Y, Han F, Yu J and Feng L: Oxymatrine ameliorates agomelatine-induced hepatocyte injury through promoting proteasome-mediated CHOP degradation. Biomed Pharmacother 114: 108784, 2019.

(c) (i) () $($ This work is licensed under a Creative Commons cc) Attribution-NonCommercial-NoDerivatives 4.0 International (CC BY-NC-ND 4.0) License. 